\title{
Crystallography of silicon element: stable and metastable crystalline forms
}

\author{
Alexandre Courac (Kurakevych) \\ IMPMC - Sorbonne University, Paris, France; \\ alexandre.courac@sorbonne-universite.fr
}

HP research on Si started more than 50 years ago and since then several allotropes, displaying a wide variety of physical properties, have been reported. The narrow-bandgap semiconductorSi-III with BC8 structure (originally believed to be semimetal) can be obtained from the high-pressure tetragonal metallic phase, Si-II, formed during compression of common silicon according to $\mathrm{Si}-\mathrm{I} \rightarrow \mathrm{Si}$ II. Such a transformation during decompression can be either direct, Si-II $\rightarrow$ Si-III, or with an intermediate step Si-II $\rightarrow$ Si-XII $\rightarrow$ SiIII. Our in situ studies of pure $\mathrm{Si}$ in oxygen-free environment indicated that in the absence of pressure medium, Si-I remains metastable at least up to $\sim 14 \mathrm{GPa}$, while the pressure medium allows reducing the onset pressure of transformation to $\sim 10 \mathrm{GPa}$. Upon heating Si-III at ambient pressure a hexagonal structure, named Si-IV, was observed. This allotrope was believed to be a structural analogue of the hexagonal diamond found in meteorites (called also lonsdaleite) with the $2 \mathrm{H}$ polytypestructure. Calculations have predicted several hexagonal polytypes of $\mathrm{Si}$ and of other Group-IV elements to be metastable, such as $2 \mathrm{H}(\mathrm{AB}), 4 \mathrm{H}(\mathrm{ABCB})$ and $6 \mathrm{H}(\mathrm{ABCACB})$. Exhaustive structural analysis, combining fine-powder X-ray and electron diffraction, afforded resolution of the crystal structure. We demonstrate that hexagonal $\mathrm{Si}$ obtained by high-pressure synthesis correspond to $\mathrm{Si}-4 \mathrm{H}$ polytype (ABCB stacking), in contrast with $\mathrm{Si}-2 \mathrm{H}$ (AB stacking) proposed previously. The sequence of transformations $\mathrm{Si}-\mathrm{III} \rightarrow \mathrm{Si}-\mathrm{IV}(4 \mathrm{H}) \rightarrow \mathrm{Si}-\mathrm{IV}(6 \mathrm{H})$ has been observed in situ by powder X-ray diffraction. This result agrees with prior calculations that predicted a higher stability of the $4 \mathrm{H}$ form over $2 \mathrm{H}$ form. Further physical characterization, combining experimental data and ab-initio calculations, have shown a good agreement with the established structure. Strong photoluminescence emission was observed in the visible region, for which we foresee optimistic perspectives for the use of this material in Si-based photovoltaics. The study of silicon allotropic transformation in Na-Si and K-Si systems at high pressure led to new open-framework allotrope of $\mathrm{Si}, \mathrm{Si} 24$ with zeolite structure and promising optoelectronic properties.

Keywords: silicon, direct bandgap material, high pressure, metastability, open framework structure 